\title{
TERT Promoter Mutation Spatial Heterogeneity in a Metastatic Follicular Thyroid Carcinoma: Implications for Clinical Work-Up
}

\author{
Adam Stenman ${ }^{1,2,3} \cdot$ Martin Hysek $^{1} \cdot$ Kenbugul Jatta $^{4} \cdot$ Robert Bränström ${ }^{2,3} \cdot$ Eva Darai-Ramqvist $^{4}$. \\ Johan O. Paulsson ${ }^{1} \cdot \mathrm{Na}^{\text {Wang }}{ }^{1} \cdot$ Catharina Larsson $^{1} \cdot$ Jan Zedenius $^{2,3} \cdot$ Carl Christofer Juhlin ${ }^{1,4}$
}

Published online: 1 June 2019

(C) The Author(s) 2019

\begin{abstract}
Follicular thyroid carcinoma (FTC) is not routinely diagnosed by a preoperative fine needle aspiration biopsy (FNAB), and the final diagnosis relies on histopathological criteria visible upon microscopic examination of the excised tumor. Several markers have been proposed as helpful in the identification of follicular thyroid tumors with malignant potential and worse prognosis, of which the specific point mutations C250T and C228T in the Telomerase Reverse Transcriptase (TERT) promoter region seem to be particularly promising. We describe a patient presenting with a large pelvic mass, in which a core needle biopsy was consistent with follicular-patterned thyroid tissue positive for a Q61R NRAS mutation and the C228T TERT promoter mutation. Upon clinical investigation, a 60-mm lesion was detected in the right thyroid lobe. The ensuing FNAB was consistent with a follicular thyroid tumor, Bethesda IV, positive for the same NRAS mutation and both the C228T and C250T TERT promoter mutations. A total thyroidectomy was performed, and a widely invasive FTC was diagnosed. Tumor tissue samples from various parts of the primary lesion were investigated for TERT promoter mutations, displaying C228T in three samples and C250T in one. Interestingly, the $\mathrm{C} 228 \mathrm{~T}$ mutations showed a coupling to areas with high $\mathrm{Ki}-67$ proliferation indexes. Our data indicate that TERT promoter mutations can exhibit spatial heterogeneity in FTCs, with implications for clinical management as well as providing insights into the molecular biology underlying the tumoral etiology.
\end{abstract}

Keywords TERT $\cdot$ Thyroid cancer $\cdot$ Metastasis $\cdot$ Mutation $\cdot$ Spatial heterogeneity

\section{Introduction}

Two somatic transversions of the Telomerase reverse transcriptase (TERT) gene promoter (chr 5:1,295,228 C $>$ T; termed C228T and chr5:1,295,250 C>T; C250T) are recurrently demonstrated in poorly differentiated thyroid carcinoma

Electronic supplementary material The online version of this article (https://doi.org/10.1007/s12022-019-09580-7) contains supplementary material, which is available to authorized users.

Carl Christofer Juhlin

christofer.juhlin@ki.se

1 Department of Oncology-Pathology, BioClinicum J6:20, Visionsgatan 4, SE-17164 Solna, Sweden

2 Department of Molecular Medicine and Surgery, Karolinska Institutet, Stockholm, Sweden

3 Department of Breast, Endocrine Tumors and Sarcoma, Karolinska University Hospital, Stockholm, Sweden

4 Department of Pathology and Cytology, Karolinska University Hospital, Stockholm, Sweden and anaplastic thyroid carcinoma, but also occur in poorprognosis cases of well-differentiated thyroid carcinomas (papillary thyroid carcinoma (PTC), and follicular thyroid carcinoma (FTC) [1]. TERT encodes the catalytic subunit of telomerase, an enzyme providing immortalization though the maintenance of telomere length, a fundamental hallmark of malignant tumors. The $\mathrm{C} 228 \mathrm{~T}$ and $\mathrm{C} 250 \mathrm{~T}$ promoter mutations alter the transcription output of the TERT gene, thereby thought to confer the tumor with a selective growth advantage.

TERT promoter mutational screening has been shown to reveal malignant potential of follicular tumors of uncertain malignant potential (FT-UMPs) and identify patients with PTC and FTC at risk for recurrence [1-7], suggesting that the implementation of this analysis is an effective method complementing routine analyses when diagnosing thyroid tumors. Even so, little is known regarding spatial distribution of these mutations within a unique lesion, and if multi-regional sampling affects the sensitivity of the method. We here present evidence of spatial heterogeneity regarding TERT promoter mutations, and emphasize the clinical implications drawn from the results. 


\section{Case Report}

The patient is a 56-year old, clinically euthyroid female of Spanish ethnicity without family history of thyroid disease. Twenty years ago, she was investigated for a thyroid nodule (lobe not specified) abroad, with benign cytology according to the patient. In December 2018, she presented with lower back pain and a subsequent $\mathrm{CT}$ scan visualized a $60-\mathrm{mm}$ large lesion in conjunction to the left sacroiliac joint adjoined by lytic lesions of the sacrum and the lower vertebrae (Supplementary Fig. 1). A core-needle biopsy demonstrated follicularpatterned cells with monomorphic nuclei without PTCassociated changes. Immunohistochemistry demonstrated positivity for TTF1, PAX8, and thyroglobulin, raising the suspicion of the lesion being metastatic with an assumed primary location in the thyroid. Molecular genetic analyses using the targeted exome-sequencing platform Oncomine Solid Tumor Panel (Ion Torrent S5, Thermo Scientific, MA, USA) and DNA extracted from formalin-fixated paraffin-embedded core-needle biopsy material revealed a Q61R NRAS mutation, and separate Sanger sequencing of the TERT promoter region revealed the C228T mutation, strengthening the suspicion of metastatic FTC. Clinical evaluation including a neck MRI and ultrasonography revealed an enlarged right thyroid lobe $(5 \times$ $6 \times 6.5 \mathrm{~cm}$ ) with a heterogeneous and tumorous appearance. A fine needle aspiration biopsy (FNAB) showed a follicular thyroid tumor, Bethesda IV, positive for the same NRAS mutation, but surprisingly displaying both C228T and C250T TERT promoter mutations (Fig. 1). Although the dissimilar TERT promoter mutation profiles could imply that the follicular thyroid tumor and the pelvic lesion were unrelated, the common denominator of NRAS mutations argued in favor of an FTC with a synchronous metastatic deposit. The patient underwent a total thyroidectomy upfront, and the pathology examination revealed a $60-\mathrm{mm}$ large, heterogenous tumor with multiple areas displaying capsular and vascular invasion compatible with a widely invasive FTC (pT3aNXM1, R0). Four different areas of the primary tumor were submitted for TERT promoter sequencing, revealing three areas with the C228T mutation and one area with the $\mathrm{C} 250 \mathrm{~T}$ mutation (Fig. 1). The Ki-67 proliferation indexes were $8 \%, 8.1 \%$, and $8.5 \%$ for the $\mathrm{C} 228 \mathrm{~T}$ areas and $2.9 \%$ for the $\mathrm{C} 250 \mathrm{~T}$ area (Fig. 1).
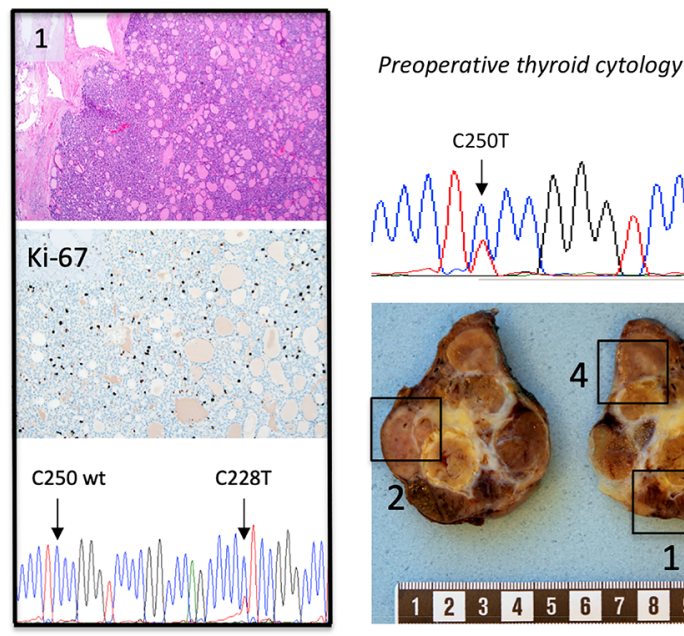

Preoperative thyroid cytolog
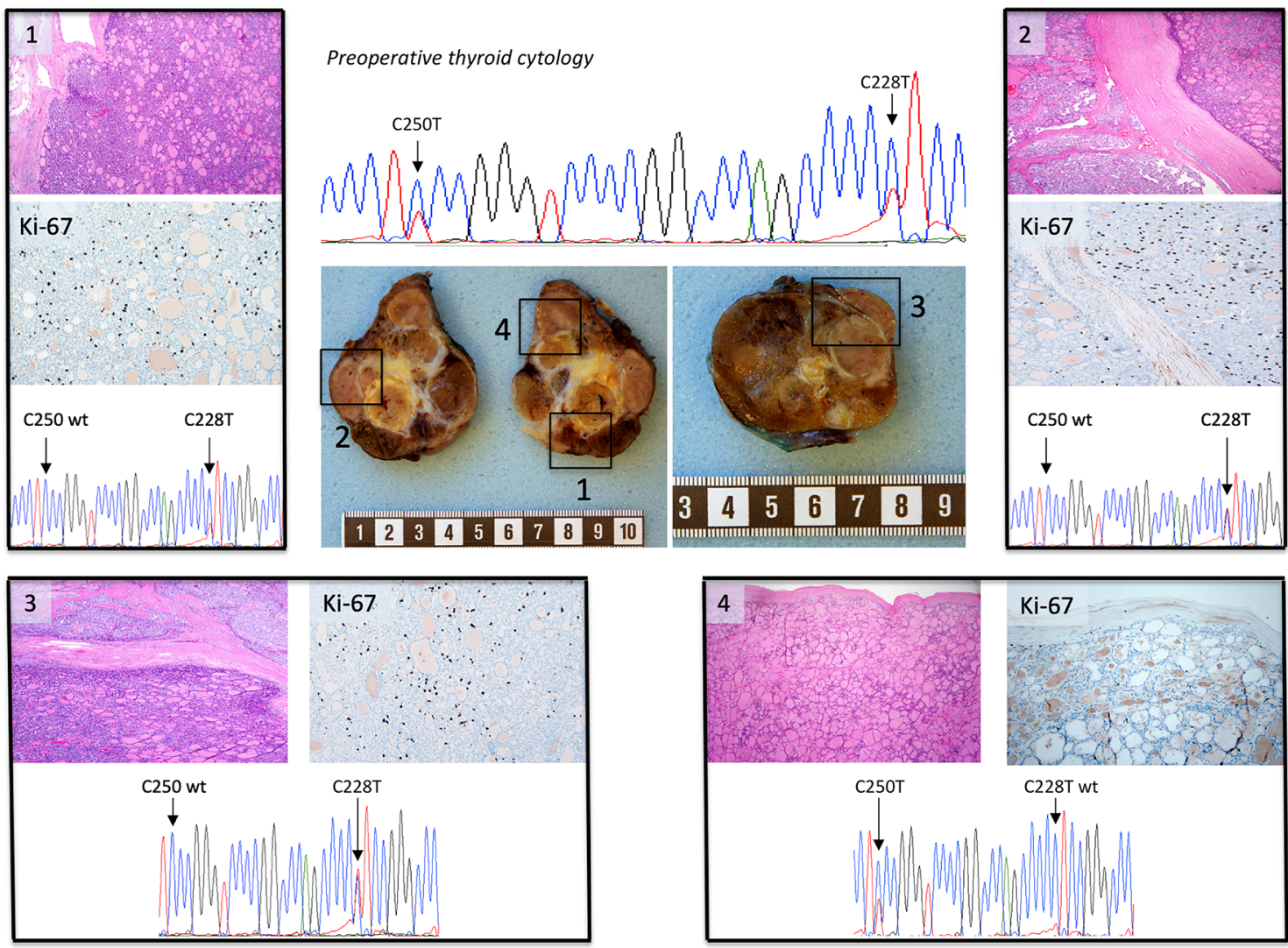

Fig. 1 Gross, microscopic, and molecular findings of the follicular thyroid carcinoma specimen. The central images depict gross findings of a follicular tumor with heterogeneous appearance, with multinodular, solid, yellow-brownish cut surface with remnants of a capsular structure. Measurement bar in centimeters. Each black square (1-4) depicts area chosen for Sanger sequencing of the TERT promoter, and each of these regions are represented with routine histology (hematoxylin-eosin

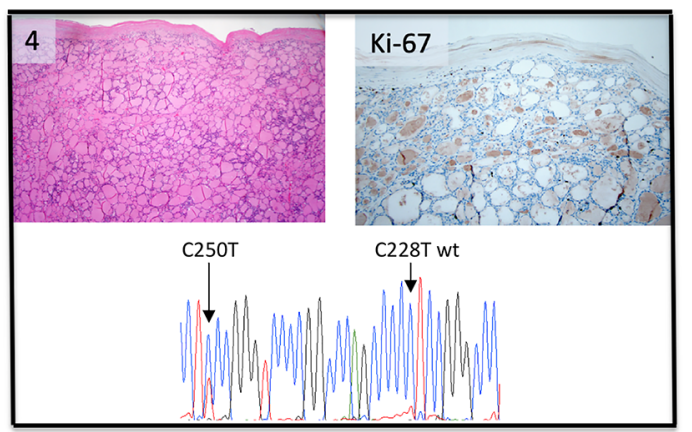

staining), a Ki-67 proliferation index as well as a chromatogram depicting the TERT promoter region including positions C228 and C250. Note how samples 1-3 have a dense, micro-follicular pattern, elevated Ki-67 scores and display the $\mathrm{C} 228 \mathrm{~T}$ mutation, while sample 4 is less cell-rich, has a lower Ki-67 index and exhibits the C250T mutation. The top central chromatogram is a representation of the preoperative cytological examination, displaying the $\mathrm{C} 228 \mathrm{~T}$ and $\mathrm{C} 250 \mathrm{~T}$ TERT promoter mutations 


\section{Discussion}

TERT promoter mutational screening is rapidly gaining ground as a confident rule-in marker of malignant properties in follicular thyroid tumors, as the occurrence of a mutation is a strong predictor of future recurrences and worse patient outcome. Moreover, as TERT immunohistochemistry displays poor correlation to overall mRNA expression in FTCs, mutational screening remains the most clinically consistent method to detect cases with aberrancies in the TERT axis [8]. In all, TERT promoter mutational screening should be considered as a reliable complimentary diagnostic marker in equivocal FTUMP cases as well as a prognostication tool for FTCs in general $[2,4]$. Until now, TERT promoter mutations have not been considered as subclonal events in thyroid cancer, and no clear guidelines as to how extensive the mutational testing should be in terms of numbers of tissue blocks submitted for analysis exist. As the TERT promoter mutations were spatially heterogeneous in this FTC, this indicates expansions of specific $\mathrm{C} 228 \mathrm{~T}$ and $\mathrm{C} 250 \mathrm{~T}$ clones that could merit further attention regarding the underlying molecular biological mechanisms driving metastatic spread. Indeed, similar observations have been made in meningioma [9]. In our case, the initial finding of dissimilar mutations generated an uncertainty regarding the relation between the thyroid tumor and the pelvic lesion, as the fact that FTCs can harbor both the C228T and C250T mutations was not obvious at the time of diagnosis. The notion that FTCs might display a spatial heterogeneity regarding these mutations is therefore of clinical importance.

In the primary tumor, we observed an association between high proliferation and the $\mathrm{C} 228 \mathrm{~T}$ mutation. Given the presence of this mutation also in the metastatic lesion, it is likely that the metastasis originated from one of these areas with high Ki-67 proliferation index. As local differences in TERT promoter mutational status could affect the interpretation of the analysis, a multi-regional sampling methodology or sampling of areas with high Ki-67 indexes could in theory increase the accuracy of the method.

We conclude that TERT promoter mutations can exhibit spatial heterogeneity in FTCs, providing insights to molecular underpinnings as well as clinical management of the disease.

Funding Information The study was supported by grants from the Swedish Cancer Society, the Stockholm County Council and the Cancer Research Funds of Radiumhemmet.

\section{Compliance with Ethical Standards}

Conflict of Interest The authors declare that they have no conflict of interest.
Ethical Statement Ethical approval has been approved by the local ethical committee, and informed consent has been obtained.

Open Access This article is distributed under the terms of the Creative Commons Attribution 4.0 International License (http:// creativecommons.org/licenses/by/4.0/), which permits unrestricted use, distribution, and reproduction in any medium, provided you give appropriate credit to the original author(s) and the source, provide a link to the Creative Commons license, and indicate if changes were made.

\section{References}

1. Liu R, Xing M (2016) TERT promoter mutations in thyroid cancer. Endocr Relat Cancer 23:R143-R155 .

2. Hysek M, Paulsson JO, Wang N, Jatta K, Lindh C, Fuentes-Martinez N, Shabo I, Zedenius J, Juhlin CC (2018) TERT promoter mutational screening as a tool to predict malignant behaviour in follicular thyroid tumours-three examples from the clinical routine. Virchows Arch 473:639-643 .

3. Cracolici V, Kadri S, Ritterhouse LL, Segal JP, Wanjari P, Cipriani NA (2019) Clinicopathologic and Molecular Features of Metastatic Follicular Thyroid Carcinoma in Patients Presenting With a Thyroid Nodule Versus a Distant Metastasis. Am J Surg Pathol 43:514-522 .

4. Paulsson JO, Mu N, Shabo I, Wang N, Zedenius J, Larsson C, Juhlin CC (2018) TERT aberrancies: a screening tool for malignancy in follicular thyroid tumours. Endocr Relat Cancer 25:723-733 .

5. Landa I, Ganly I, Chan TA, Mitsutake N, Matsuse M, Ibrahimpasic T, Ghossein RA, Fagin JA (2013) Frequent somatic TERT promoter mutations in thyroid cancer: higher prevalence in advanced forms of the disease. J Clin Endocrinol Metab 98:E1562-E1566.

6. Liu X, Bishop J, Shan Y, Pai S, Liu D, Murugan AK, Sun H, ElNaggar AK, Xing M (2013) Highly prevalent TERT promoter mutations in aggressive thyroid cancers. Endocr Relat Cancer 20:603610 .

7. Xing M, Liu R, Liu X, Murugan AK, Zhu G, Zeiger MA, Pai S, Bishop J (2014) BRAF V600E and TERT promoter mutations cooperatively identify the most aggressive papillary thyroid cancer with highest recurrence. J Clin Oncol 32:2718-2726 .

8. Paulsson JO, Olander A, Haglund F, Zedenius J, Juhlin CC (2018) TERT Immunohistochemistry Is a Poor Predictor of TERT Promoter Mutations and Gene Expression in Follicular Thyroid Carcinoma. Endocr Pathol 29:380-383 .

9. Juratli TA, Thiede C, Koerner MVA, Tummala SS, Daubner D, Shankar GM, Williams EA, Martinez-Lage M, Soucek S, Robel K, Penson T, Krause M, Appold S, Meinhardt M, Pinzer T, Miller JJ, Krex D, Ely HA, Silverman IM, Christiansen J, Schackert G, Wakimoto H, Kirsch M, Brastianos PK, Cahill DP (2017) Intratumoral heterogeneity and TERT promoter mutations in progressive/higher-grade meningiomas. Oncotarget 8:109228109237 .

Publisher's Note Springer Nature remains neutral with regard to jurisdictional claims in published maps and institutional affiliations. 